\title{
Elastic Energy Loss in an Expanding QGP
}

\author{
M. B. Gay Ducati, \\ Instituto de Física, Universidade Federal do Rio Grande do Sul \\ Av. Bento Gonçalves 9500, \\ Campus do Vale, Caixa Postal 15051 \\ CEP 91501-970, Porto Alegre, RS, Brazil \\ V. P. Gonçalves, \\ Instituto de Física e Matemática - Universidade Federal de Pelotas \\ Campus Universitário s/n - Caixa Postal 354 \\ CEP 96010-900, Pelotas, RS, Brazil \\ and L. F. Mackedanz \\ Centro de Ciências Exatas e Tecnológicas - Universidade Federal do Pampa/UFPel \\ Campus Caçapava do Sul - Rua Rui Vieira Machado s/n \\ CEP 96570-000, Caçapava do Sul, RS, Brasil \\ Instituto de Física - Universidade Federal do Rio Grande do Sul \\ Av. Bento Gonçalves 9500 - Campus do Vale - Caixa Postal 15051 \\ CEP 91501-970, Porto Alegre, RS, Brazil
}

Received on 21 January, 2007

\begin{abstract}
The discovery of the jet quenching in central $\mathrm{Au}+\mathrm{Au}$ collisions at the Relativistic Heavy-ion Collider (RHIC) has provided clear evidence for the formation of strongly interacting dense matter. It has been predicted to occur due to the energy loss of high energy partons that propagate through the quark gluon plasma. Since the medium is not static and it cools while expands, the strong coupling is not fixed, running with the evolution of the system. In this work, we present an investigation of the dependence on the value of the strong coupling in the parton energy loss due to elastic scatterings in a parton plasma. We analyze different prescriptions for the QCD coupling and calculate the energy and length dependence of the fractional energy loss. Moreover, the quenching factor for light and heavy quarks is estimated. We found that the predicted enhancement of heavy to light hadrons $(D / \pi)$ ratio is strongly dependent on the running of the QCD coupling constant.
\end{abstract}

Keywords: Quark Gluon Plasma; Elastic energy loss; Running coupling constant

In the last years, the understanding of the partonic energy loss has been extensively developed. It was provocated by the expectation of the Quark Gluon Plasma (QGP) formation in the early stages of the collision. The discovery of jet quenching in central Au+Au collisions at RHIC has provided evidences for the formation of this strongly interacting dense matter. Basically, high parton density produced in heavy ion collisions could induce a large amount of energy loss for hard partons produced in the initial stage of the collision during its propagation through the fireball, due to their interactions with the medium.

The total energy loss of a particle in a medium can be decomposed into an elastic and a radiative contribution. At large energies one expects that radiative energy loss becomes much larger than the collisional one, as in the electromagnetic case. However, since at lower energies the elastic and radiative processes can contribute equally, for small values of the parton energy the collisional one can dominate. So, an open question is to quantify the contribution of each process in the RHIC kinematical region. Recent studies of elastic mechanism contributions to energy loss [1-4] have indicate that, for RHIC regimes, it is far from clear that radiative energy loss dominates over elastic one.

Besides, we have to consider that the medium properties and interactions change with time (For a discussion of the medium evolution see, e.g. Refs. [5, 6]), since in nucleusnucleus collisions, at collider energies, the produced hard partons propagate through a rapidly expanding medium. The scenario assumed here consider that the interactions in the plasma of quarks and gluons get stronger with the time because the average parton energies drop due to the expansion of the system $[7,8]$. The basic idea comes from the feature that the QGP is not a static medium, but it is cooling while partons propagate through. We assume that the temperature is the dominant scale and consequently will control the running of the QCD coupling. As the temperature drops over the lifetime of the QGP, $\alpha_{s}$ should also vary during the equilibration and the evolution of the plasma. Furthermore, two prescriptions for the temperature dependence of the running coupling constant was considered. We denote them as thermal $\left[\alpha_{s}^{(t h)}\right]$, which

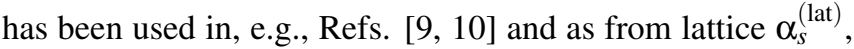
obtained from recent results in the lattice [11].

As the temporal development of the coupling constant directly influences the various particle signatures [7, 8, 12-14], we can expect a similar effect in the estimates of the energy loss of a parton propagating in a QGP. So we estimate [3] the influence of the running coupling constant in the elastic energy loss, presenting a reanalyzes of the studies from Refs. $[1,2,15,16]$, and an estimate of the quenching factor $Q\left(p_{\perp}\right)$ of light and heavy quarks. 
Since the parton interacts with the medium during its propagation through it, after escaping the collision the parton will have lost an energy fraction $\Delta E$. Consequently, the inclusive transverse momentum spectra of the particles produced in nucleus - nucleus collisions will be modified with respect to hadron - hadron collisions. In general the hadron formation is described in terms of parton recombination and/or by the fragmentation of the energetic partons. In particular, it is expected that for $p_{\perp}>5 \mathrm{GeV}$ the hadrons are dominantly produced by fragmentation. So, we will assume that fragmentation is the dominant process of hadron formation and that it occurs after the parton has left the comoving medium. It allows to analyze the energy loss effects directly in the $p_{\perp}$ spectrum of the scattered partons. We can express these effects in the computation of the quenching factor, following the assumption [17]

$$
Q\left(p_{\perp}\right)=\frac{d N^{\mathrm{med}}}{d^{2} p_{\perp}} / \frac{d N^{\mathrm{vac}}}{d^{2} p_{\perp}}
$$

where

$$
\frac{d N^{\mathrm{med}}}{d^{2} p_{\perp}}=\frac{1}{2 \pi^{2} R^{2}} \int_{0}^{2 \pi} d \phi \int_{0}^{R} d^{2} r \frac{d N^{\mathrm{vac}}\left(p_{\perp}+\Delta E\right)}{d^{2} p_{\perp}}
$$

and $\Delta E$ is the total energy loss by partons in the medium. Moreover, $Q\left(p_{\perp}\right)$ is the medium dependent quenching factor. For a realistic calculation of the quenching, the knowledge of the full probability distribution is actually required [18].

In order to calculate $\Delta E$ we will use the approach proposed by Svetitsky in Ref. [15], which considers the Brownian motion of a parton in a thermal bath, governed by the FokkerPlanck equation. In this approach one starts from the Boltzmann equation for the distribution function $f(x, p)$, which in the relativistically covariant form can be written as

$$
p^{\mu} \partial_{\mu} f(x, p)=C\{f\},
$$

where $p^{\mu}=\left(E_{\vec{p}}, \vec{p}\right)$ is the four-momentum of the test quark and $f$ is its phase-space density and $C\{f\}$ is the collision term. Following Refs. [1, 2, 15, 16] we assume that: (a) the hydrodynamical evolution can be described by the Bjorken scenario, which implies that it is valid to assume that the plasma is uniform and consequently the phase space density of the quark is independent of $\vec{x}$ and (b) the collision term is given by the elastic collisions of the test quark with other quarks, antiquarks and gluons in the system. Using the Landau approximation and restringing the analyzes to the one-dimensional problem one obtain $[1,2,15,16]$ the Landau kinetic equation.

If we consider that the background heat bath is constituted of a large amount of weakly coupled particles in thermal equilibrium at a temperature $\mathrm{T}$, with some non-thermal but homogeneously distributed particles due to the fluctuations, the problem can be simplified assuming that the equilibrium of the bath will not be disturbed by the presence of these few nonthermal particles. Due to their small number, one can also assume that they will not interact among themselves, only with particles of the thermal bath. So, one can replace the distribution functions of the collision partners of the test particle by their Fermi-Dirac or Bose-Einstein distributions and the Landau kinetic equation reduces to the Fokker-Planck equation, which is expressed as follows $[1,2,15,16]$

$$
\frac{\partial f}{\partial t}=\mathcal{A} \frac{\partial}{\partial p}(p f)+\mathcal{D}_{F} \frac{\partial^{2} f}{\partial p^{2}}
$$

where $\mathcal{A}$ is the drag coefficient and $\mathcal{D}_{F}$ is the diffusion coefficient, which is given by $\mathcal{D}_{F}=\mathcal{A} T^{2}$ if we assume that the momentum $p$ can be approximated by the temperature $T$ of the system and the coupling between the Brownian particle and the bath is weak. The Eq. (4) describes the evolution of the momentum distribution of a test particle undergoing Brownian motion. The Gaussian shaped solution from Eq. (4) has been obtained in Refs. [1, 2], assuming as the boundary condition $f(p, t) \stackrel{t \rightarrow t_{0}}{\longrightarrow} \delta\left(p-p_{0}\right)$ and using the method of characteristics $[1,2]$

Then, we can estimate the mean energy of the parton due to elastic collisions, after traversing a distance $L$ as

$$
\langle E\rangle=\int_{0}^{\infty} E f(p, L) d p
$$

where $f(p, L)$ is the solution of Eq. (4) and the average energy loss due to elastic collisions in the medium will be given by

$$
\Delta E=E_{0}-\langle E\rangle
$$

where $E=m_{\perp}=\sqrt{p_{\perp}^{2}+M^{2}}$ at the central rapidity region, $y=0$. Consequently, in order to estimate the average energy loss it is necessary to solve Eq. (4). The solution of Eq. (4) is strongly dependent on the drag coefficient. Following Refs. $[15,16]$, we approximate the drag coefficient by its average value,

$$
\langle\mathcal{A}(p, t)\rangle=\mathcal{A}(t)=\left\langle-\frac{1}{p} \frac{d E}{d L}\right\rangle
$$

which is directly dependent of the energy loss rate $d E / d L$. The above approximation is reasonable up to moderate momentum values ( $p \leq 15 \mathrm{GeV})[2]$.

The energy loss rate in the QGP due to elastic collisions with high-momentum transfer have been originally estimated by Bjorken [20] and recalculated in Refs. [21-24] taking into account the loss with low-momentum transfer dominated by the interactions with plasma collective modes in the hard thermal loop approximation [25]. In particular, in Ref. [23] the authors have estimated the energy loss for heavy quarks and in Ref. [22] for light partons. For heavy quarks, it reads

$$
-\frac{d E}{d L}=\frac{8 \pi \alpha_{s}^{2} T^{2}}{3}\left(1+\frac{n_{f}}{6}\right)\left[\frac{1}{v}-\frac{1-v^{2}}{2 v^{2}} \ln \left(\frac{1+v}{1-v}\right)\right] \ln \left[2^{\frac{n_{f}}{6+n_{f}}} B(v) \frac{E T}{m_{g} M}\right] \quad \text { for } E<<M^{2} / T
$$




$$
-\frac{d E}{d L}=\frac{8 \pi \alpha_{s}^{2} T^{2}}{3}\left(1+\frac{n_{f}}{6}\right) \ln \left[2^{\frac{n_{f}}{2\left(6+n_{f}\right)}} 0.92 \frac{\sqrt{E T}}{m_{g}}\right] \quad \text { for } E>>M^{2} / T
$$

where $n_{f}$ is the number of quark flavors, $\alpha_{s}$ is the strong coupling constant, $m_{g}=\sqrt{\left(1+n_{f} / 6\right) g^{2} T^{2} / 3}$ is the thermal gluon mass, $E$ is the energy and $M$ is the mass of the quark. $B(v)$ is a smooth velocity function, which can be taken approximately as 0.7 [23]. For light quarks we use the expression (9) and set $M=0$ in the calculations. At the energies (temperatures)

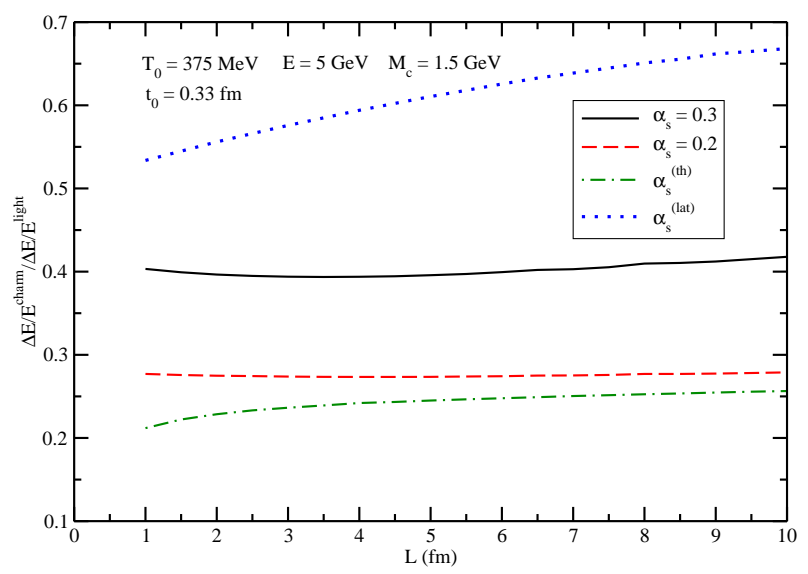

FIG. 1: Heavy-to-light ratio of the fractional energy loss

which we are interested in this paper the $\operatorname{drag}$ coefficient $\mathcal{A}$ for partons propagating in a plasma can be calculated using Eq. (7) and the expressions for elastic energy loss given by Eqs. (8) and (9). The average over the momentum is made using the Boltzmann distribution. In order to simplify our considerations we model the space-time evolution of the quark-gluon plasma by the Bjorken scenario with boost invariant longitudinal expansion and conserved entropy per rapidity unit [19] and neglect the transverse expansion of the system. Assuming this scenario, the time dependence of the temperature is given by $T(t)=t_{0}^{1 / 3} T_{0} / t^{1 / 3}$, where $t_{0}$ and $T_{0}$ are, respectively, the initial time and temperature at which the background of the partonic system has attained local kinetic equilibrium. The time dependence from the drag coefficient is directly associated with this evolution for the temperature, which decreases with time as the system expands. We assume as maximum time limit for the evolution the length of the plasma $L$. These approximations has been considered in Refs. $[1,2]$ which we would like compare our results. Moreover, we assume $T_{0}=375 \mathrm{MeV}$ and $t_{0}=0.33 \mathrm{fm}$ for RHIC energies.

In Fig. 1 we present the ratio between the fractional energy loss for heavy and light quarks. While for fixed $\alpha_{s}$ the ratio is almost constant in the range considered, for running $\alpha_{s}$ with the lattice prescription, the ratio is monotonously increasing, and the value is greater than 0.5 in all the range considered;

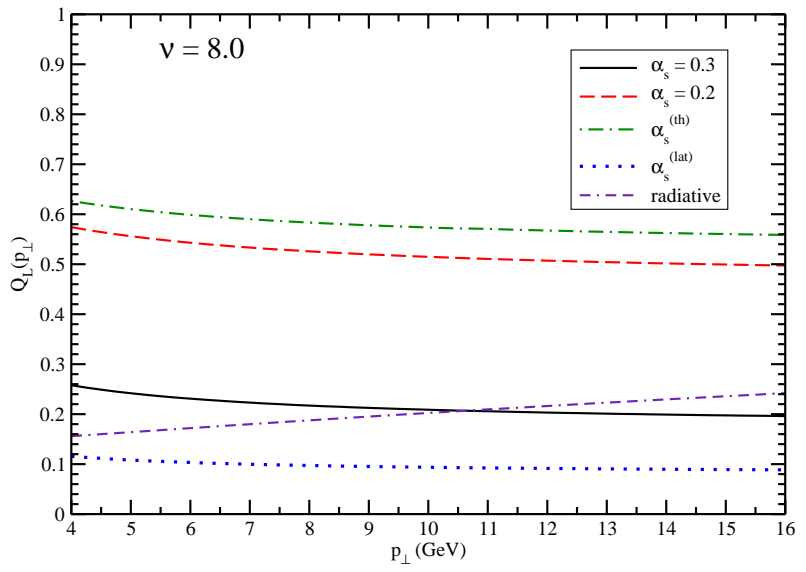

FIG. 2: Quenching factor for light quarks

on the other hand, with the thermal prescription, the ratio is strongly suppressed when compared with the result for fixed $\alpha_{S}$. This feature suggests that the heavy quarks lose less than $20 \%$ of the energy lost by light quarks in its path through the fireball.

In order to compute the $p_{\perp}$-spectra, we assume that the geometry is described by a cylinder of radius $R$ and the parton moves in the transverse plane in the local rest frame. Furthermore, we will assume the following parameterization of the $p_{\perp}$ distribution

$$
\frac{d N_{L}^{\mathrm{vac}}}{d^{2} p_{\perp}}=A\left(\frac{1}{p_{0}+p_{\perp}}\right)^{v}
$$

where $v=8.0$ and $p_{0}=1.75 \mathrm{GeV}$ [17]. The results for the quenching factor for light quarks are shown in the Fig. 2 for both sets of parameters. For a comparison, we present a estimative of the quenching due to the radiative energy loss, following the parameterization proposed in Ref. [17]. Due to the smaller drag coefficient, the thermal QCD $\alpha_{S}$ prescription gives a high quenching factor, so the spectrum is less modified by collisional energy loss than in the case of fixed $\alpha_{S}$. At $\alpha_{S}=0.3$ we have that in the high $p_{\perp}$ region elastic and radiative energy loss are of the same order of magnitude. On the other hand, the lattice QCD $\alpha_{S}$ prescription implies a very large modification of the spectrum. Finally, if the system presents a lower value of $\alpha_{S}$ than considered in earlier calculations, the gluon bremsstrahlung becomes the dominant mechanism of energy loss again.

For heavy quarks, we use the $p_{\perp}$ distribution of charmed hadrons, $D$-mesons, produced in hadron collisions, experimentally found [27] to be well described by the following 


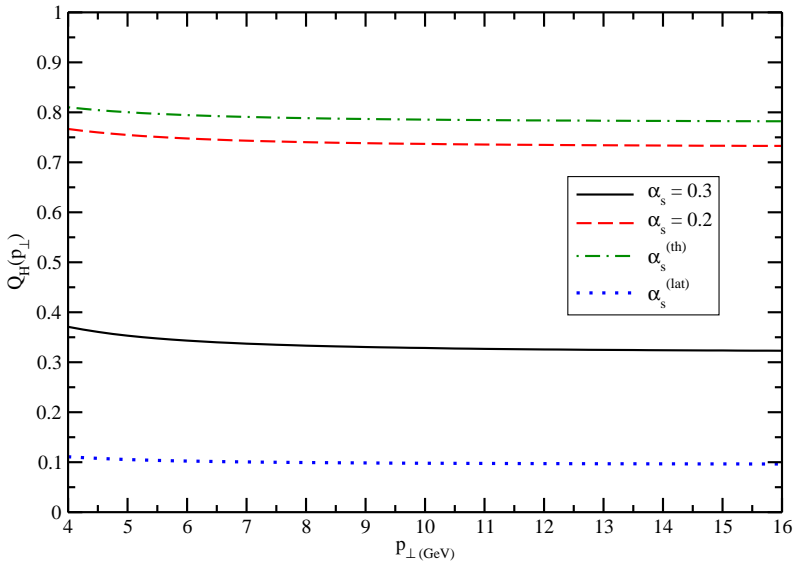

FIG. 3: Quenching factor for charm quarks

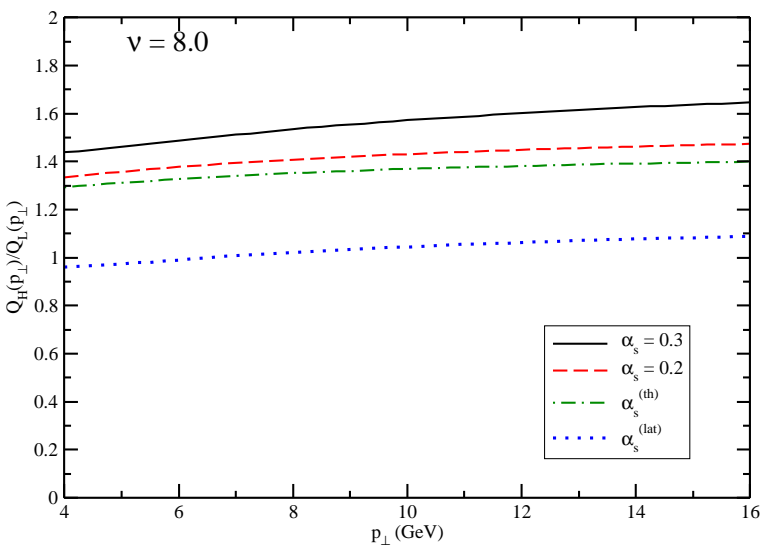

FIG. 4: Charm-to-light ratio of quenching factors

simple parameterization as

$$
\frac{d N_{H}^{\mathrm{vac}}}{d^{2} p_{\perp}}=C\left(\frac{1}{b M_{c}^{2}+p_{\perp}^{2}}\right)^{n / 2},
$$

where $b=1.4 \pm 0.3, n=10.0 \pm 1.2$ and $M_{c}=1.5 \mathrm{GeV}$. Eq. 11 was fitted from fixed target data and its validity at RHIC energies could be questionable. However, since our goal is the study of quenching ratios and their behaviour with the changes of the medium properties, we use Eq. 11 to simplify our calculation. The quenching factor for heavy quarks is shown in Fig. 3. The results are similar to the case of light quarks, presented in Fig. 2. The thermal QCD $\alpha_{S}$ prescription gives the higher quenching factor, and the lattice QCD one gives the smaller factor, shown a strong suppression in the charm spectra when this prescription is used. Again, smaller is the $\alpha_{S}$ value, higher the quenching factor due to elastic scattering in the QGP.

Recently, the ratio between the spectra of hadrons with heavy quarks and with light quarks has been proposed as a tool to investigate the medium formed in heavy ion collisions [26]. Because of their large mass, radiative energy loss for heavy quarks would be lower than for light quarks. The predicted consequence of this distinct radiative energy losses is an enhancement of this ratio at moderately large transverse momentum, relative to that observed in the absence of energy loss (A recent analysis for LHC energies is given in Ref. [28]). As the behavior of this ratio considering collisional energy loss is still an open question, in Fig. 4 we present the ratio between heavy and light quark quenching factors, which reflects the heavy to light hadrons $(D / \pi)$ ratio, considering only collisional energy loss. While for fixed $\alpha_{S}$, the results show an enhancement factor close to 1.4 , this enhancement is suppressed when running coupling prescriptions are considered. This feature could suggest that with an expanding cooling medium, the collisional energy loss for heavy and light quarks would be of similar magnitude.

As a summary, we have investigated the dependence of the parton collisional energy loss in a QGP on the value of the strong coupling. Since the plasma is not a static medium, a fixed value for $\alpha_{s}$ had to be treated as an approximation, and more realistic estimatives should consider the evolution of the fireball. We have considered running coupling in the calculation, evolving it with the cooling of the QGP. From the Fokker-Planck equation, we derived the transport coefficients and related them with parton mean energy loss. For light quarks, we found that the radiative and collisional energy loss are of the same order of magnitude, in the high $p_{\perp}$ region, if the $\alpha_{S}$ value is compared or greater than 0.3 . For smaller values of coupling, the gluon bremsstrahlung becomes the dominant process for energy loss, again. We also presented the ratio between heavy and light quenching factors, and found an absence of enhancement of the heavy to light hadrons ratio if running coupling is used. It was a striking result, since it seems to suggest that heavy and light quarks have the same order of magnitude of collisional energy loss. Our results motivate a similar study in radiative parton energy loss.

Ackowledgements. This work was partially financed by CNPq and FAPERGS, Brazil.
[1] M. G. Mustafa and M. H. Thoma, Acta Phys. Hung. A 22, 93 (2005) [arXiv:hep-ph/0311168].

[2] M. G. Mustafa, Phys. Rev. C 72, 014905 (2005).

[3] M. B. Gay Ducati, V. P. Gonçalves, L. F. Mackedanz, arXiv:hep-ph/0506241

[4] A. K. Dutt-Mazumder et al., Phys. Rev. D 71, 094016 (2005).
[5] I. Vitev and M. Gyulassy, Phys. Rev. Lett. 89, 252301 (2002)

[6] C. A. Salgado and U. A. Wiedemann, Phys. Rev. Lett. 89, $092303(2002)$

[7] S. M. H. Wong, Phys. Rev. C 56, 1075 (1997).

[8] G. C. Nayak et al., Nucl. Phys. A 687, 457 (2001)

[9] J. F. Gunion, R. Vogt, Nucl. Phys. B 492, 301 (1997) . 
[10] P. Levai and R. Vogt, Phys. Rev. C 56, 2707 (1997)

[11] O. Kaczmarek et al., Phys. Rev. D 70, 074505 (2004) .

[12] J. Letessier, A. Tounsi and J. Rafelski, Phys. Lett. B 389, 586 (1996).

[13] S. M. H. Wong, Phys. Rev. C 58, 2358 (1998).

[14] D. d'Enterria, D. Peressounko, arXiv:nucl-th/0503054.

[15] B. Svetitsky, Phys. Rev. D 37, 2484 (1988) .

[16] M. G. Mustafa, D. Pal and D. K. Srivastava, Phys. Rev. C 57, 889 (1998)

[17] B. Müller, Phys. Rev. C 67, 061901(R) (2003) .

[18] R. Baier et al., JHEP 0109, 033 (2001).

[19] J. D. Bjorken, Phys. Rev. D 27, 140 (1983).
[20] J. D. Bjorken, FERMILAB-PUB-82-059-THY.

[21] S. Mrowczynski, Phys. Lett. B 269, 383 (1991).

[22] M. H. Thoma, Phys. Lett. B 273, 128 (1991).

[23] E. Braaten and M. H. Thoma, Phys. Rev. D 44, 2625 (1991).

[24] M. H. Thoma, Eur. Phys. J. C 16, 513 (2000)

[25] E. Braaten and R. D. Pisarski, Phys. Rev. Lett. 64, 1338 (1990); Nucl. Phys. B 337, 569 (1990); B 339, 310 (1990).

[26] Y. L. Dokshitzer, D. E. Kharzeev, Phys. Lett. B 519, 199 (2001).

[27] C. A. Alves et al. [E769 Collaboration], Phys. Rev. Lett. 77, 2392 (1996).

[28] A. Dainese, Eur. Phys. J. C 33, 495 (2004). 